\section{Análise epidemiológica \\ dos acidentes com material \\ biológico registrados no Centro \\ de Referência em Saúde do \\ Trabalhador - Londrina-PR}

\section{Epidemiological analysis of \\ accidents with biological material reported to the Worker's Health Reference Center in Londrina-PR}

\section{Regina Stella Spagnuolo'}

Renata Cristina Silva Baldo²

Ivan Amaral Guerrini ${ }^{3}$

${ }^{1}$ Departamento de Enfermagem. Centro de Ciências da Saúde, Universidade Norte do Paraná. Londrina, PR.

${ }^{2}$ Secretaria Municipal de Saúde. Centro de Referência em Saúde do

Trabalhador. Londrina, PR.

${ }^{3}$ Departamento de Física e Biofísica. Instituto de Biociências, UNESP. Botucatu, SP.

\section{Resumo}

Objetivo: Analisar a distribuição dos acidentes de trabalho com exposição a material biológico das fichas de notificação registradas no Centro de Referência de Saúde do Trabalhador de Londrina, traçando um perfil dos profissionais acidentados. Métodos: Trata-se de um estudo retrospectivo descritivo, com abordagem quantitativa, realizado através de um levantamento nas 253 fichas de notificação de acidentes com material biológico que foram encaminhadas das instituições de saúde da área de abrangência ao Centro de Referência em Saúde do Trabalhador do município de Londrina-PR, no período de janeiro a dezembro de 2006. Os dados foram processados e tabulados eletronicamente, utilizando-se o programa Epi Info, de domínio público. Resultados: Das 253 fichas de notificação de acidentes com material biológico analisadas, verificou-se que $92,5 \%$ foram causados por objetos perfuro-cortantes e 39,5\% ocorreram em auxiliares de enfermagem. Constatou-se também que 73,5\% eram do sexo feminino, 24,1\% ocorreram em Unidades Básicas de Saúde, $74,3 \%$ encontravam-se com situação vacinal para Hepatite B atualizada e 49,8\% ocorreram na rede pública de saúde. Conclusão: Com o levantamento do perfil dos profissionais acidentados com exposição a material biológico, o CEREST Londrina identificou que a profissão mais atingida foram os auxiliares de enfermagem, as mulheres, e que grande parte dos acidentes ocorreram nas UBSs com material perfurocortante. Mostrou-se que coletores de lixo passaram a fazer parte das profissões envolvidas nos acidentes.

Palavras-chave: Acidentes de trabalho. Exposição ocupacional. Epidemiologia. 


\section{Abstract}

Objective: The purpose of this paper is to analyze the distribution of occupational accidents involving exposure to biological materials reported to Londrina's Worker's Health Reference Center, and to establish a pattern for the accidents found. Method: This is a descriptive and retrospective study, with a quantitative analysis of 253 accidents with biological material reported. Accidents were reported by health institutions linked to Londrina's Worker's Health Reference Center from January to December 2006. Data were processed and tabulated by Epi Info free software. Results: Out of the 253 files analyzed, $92.5 \%$ of the accidents were caused by sharp objects, and $39.5 \%$ occurred with nurse assistants. In this sample, $73.5 \%$ of the accidents were with women, $24.1 \%$ occurred in Basic Health Units, $74.3 \%$ had updated Hepatitis B vaccination, and $49.8 \%$ occurred within the public healthcare system. Conclusion: Along with the description of the profile of workers who suffered accidents with exposure to biological material, this study found out that the most vulnerable healthcare workers are nurse assistants and women. Most of the accidents occurred in Basic Healthcare Units with sharp objects. We also found that garbage collectors are among the workers most often affected by accidents.

Key Words: Accidents. Occupational. Occupational Exposure. Epidemiology.

\section{Introdução}

No Brasil, as preocupações com medidas profiláticas e o acompanhamento clínicolaboratorial em relação aos trabalhadores de saúde expostos ao risco de acidentes de trabalho só se deu a partir da epidemia de infecção pelo HIV/Aids, no início da década de 80 e de forma ainda muito incipiente ${ }^{1,2}$.

Nessa mesma década desencadearamse condutas pré e pós-exposições, indicadas para prevenir o risco de exposição aos patógenos de transmissão sangüínea de profissionais de saúde pelo Vírus da Imunodeficiência Humana (HIV) e pelos vírus da hepatite $\mathrm{B}$ e $\mathrm{C}$ no ambiente de trabalho. Sabe-se que este tipo de acidente, quando ocorre, deve ser tratado como emergência médica, uma vez que para atingir maior eficácia, as intervenções necessitam ser iniciadas logo após a ocorrência deste ${ }^{1,2}$.

De acordo com a Lei no 8.213 , de 24 de julho de 1991, que dispõe sobre os Planos de Benefícios da Previdência Social, a definição de acidente de trabalho descrita em seu artigo 19 é "aquele que ocorre pelo exercício do trabalho, a serviço da empresa, ou ainda pelo serviço de trabalho de segurados especiais, provocando lesão corporal ou perturbação funcional, que cause a morte ou redução da capacidade do trabalho, permanente ou temporária. São considerados também como acidente de trabalho os acidentes de trajeto, as doenças profissionais e as doenças do trabalho"3.

Os profissionais de saúde envolvidos na dinâmica da assistência ao paciente, especialmente os de enfermagem, focados no "fazer" em saúde, são os que se encontram mais susceptíveis a esse tipo de acidente ${ }^{4,5}$. Entretanto, outros profissionais, como coletores de lixo, policiais militares, bombeiros, zeladores, motoristas de ambulâncias, têm sofrido acidentes com exposição a material biológico que, por conseqüência, geraram notificação e conduta ${ }^{6}$.

Em casos de exposição por material biológico, o Ministério da Saúde vem desenvolvendo protocolos de atendimento ao profissional exposto ao risco e determinando que os 
serviços de saúde tenham protocolos escritos com condutas claras em relação a esse tipo de acidente. Deve-se lembrar que o Ministério da Saúde, apenas na década de 1990, por meio da Coordenação Nacional de DST/Aids, preocupado com o aumento desse agravo, apresentou um manual de atendimento que contemplava e orientava condutas frente à exposição a material biológico.

Outros manuais foram editados posteriormente com finalidades de atualizar as condutas pré e pós-exposição, indicadas para prevenir o risco de contaminação de profissionais de saúde pelo vírus da Imunodeficiência Humana (HIV) e pelos vírus da Hepatite B e C no ambiente de trabalho.

No ano de 2006, um novo protocolo foi elaborado pelo Ministério da Saúde (MS) e pela Coordenação Nacional de Saúde do Trabalhador (COSAT), permitindo o atendimento aos profissionais de saúde que sofrem exposição a material biológico com risco de soroconversão $\mathrm{HIV}, \mathrm{HBV}, \mathrm{HCV}$, estabelecendo um fluxo de atendimento, tratamento e notificação de casos?

Essa conduta vem ao encontro da implantação da Portaria no $777 / \mathrm{MS}$, de 28 de abril de 2004, a qual inclui 11 agravos relacionados ao trabalho no Sistema de Informação de Agravos de Notificação (SINAN/NET), incluído entre eles o acidente com material biológico.

O conhecimento dos acidentes de trabalho com material biológico de uma determinada área geográfica pode ser o ponto de partida para a priorização do desenho de medidas de correção das condições de trabalho.

Reconhece-se que a implantação de protocolos já existentes e fluxogramas para $o$ atendimento adequado desse profissional de saúde, medidas de segurança acessível, política de revisão dos procedimentos/atividades e de educação continuada, planejamento e organização do trabalho são fatores essenciais que devem ser observados com o intuito de reduzir o índice de acidentes com material biológico.

Considerando a expressiva falta de registro e notificação dessas ocorrências no Brasil, alguns trabalhos indicam aproximadamente $50 \%$ de subnotificações dessas exposições ${ }^{8,9}$ Esses dados poderão ser avaliados mais fidedignamente a partir de janeiro de 2007 com os registros do SINAN/NET.

Concomitantemente a todas essas preocupações, preconizou-se o uso das precauções universais, atualmente denominadas de precauções básicas, que foram instituídas com base no princípio de que todo paciente deve ser considerado como potencialmente infectado, independente do diagnóstico definido ou presumido de doença infecciosa ${ }^{5,10}$.

Trata-se de medidas que devem ser utilizadas na manipulação de sangue, secreções e excreções, assim como no contato com mucosas e pele com áreas de integridade comprometida. Incluem recomendações sobre o uso de equipamentos de proteção individual (luvas, gorros, óculos, capotes) com a finalidade de reduzir a exposição do trabalhador da saúde ao sangue ou fluídos corpóreos, e cuidados específicos na manipulação e descarte de materiais perfuro-cortantes.

Interessante notar que apenas em 1995, a partir da Lei n. 8.975, de 5 de janeiro, o termo Biossegurança teve sua real significação na criação da Comissão Técnica Nacional de Biossegurança (CTNBio), tendo esta uma abrangência mais ampla, que extrapola a área da saúde e do trabalho, sendo empregada quando há referência ao meio ambiente e à biotecnologia ${ }^{11}$.

Todavia, a despeito da criação de todos os protocolos e leis, não se percebe ainda um verdadeiro empenho de todos os envolvidos nessa problemática de discutir as questões de acidentes de trabalho envolvendo o trabalhador de saúde em seu ambiente de trabalho.

O município de Londrina-PR, através da Secretaria Estadual e Municipal de Saúde, preocupado com esse agravo de saúde pública, no final de 1997 implantou o Protocolo de Atendimento a Acidentes de Trabalho com Material Biológico, recomendado pelo Ministério da Saúde, definindo 
um fluxograma de atendimento para Londrina e região, e instituiu as Unidades de Referência de atendimento 24 horas para os acidentes dessa natureza. Mais tarde, com a habilitação do Centro de Referência em Saúde do Trabalhador, esse atendimento pôde ser reavaliado e readequado, o que trouxe para o serviço um aumento das notificações devido à maior conscientização das instituições de saúde de Londrina e região metropolitana.

Este trabalho tem por objetivo analisar a distribuição dos acidentes de trabalho com exposição a material biológico proveniente das fichas de notificação registradas no CEREST-Londrina, traçando um perfil dos profissionais acidentados.

\section{Metodologia}

Trata-se de um estudo retrospectivo descritivo com abordagem quantitativa, realizada através de um levantamento nas 253 fichas de notificação de acidentes com material biológico, que foram encaminhadas das instituições de saúde da área de abrangência ao Centro de Referência em Saúde do Trabalhador do município de Londrina no período de janeiro a dezembro de 2006.

Os dados foram processados e tabulados eletronicamente utilizando o programa Epi Info versão 3.3, de domínio público.

Em seu aspecto ético, o estudo foi submetido ao Comitê de Ética e Pesquisa (CEP) conforme Resolução 196/96 do Conselho Nacional de Saúde e não apresenta conflito de interesses.

\section{Resultados e Discussões}

Na Tabela 1 é notória a freqüência com que ocorrem acidentes com material puntiforme, tais como lâminas de bisturi, scalps e agulhas, principalmente no ato recorrente de reencape. Pesquisas confirmam a alta incidência de acidentes ocorridos com perfurações o que reforça a necessidade urgente de permanente vigilância e treinamentos contínuos quanto aos cuidados na manipulação desses objetos ${ }^{12-14}$.
Tabela 1 - Distribuição dos acidentes com exposição a material biológico, segundo objeto causador. Londrina, PR, 2006.

Table 1 - Distribution of accidents with exposure to biological material by causing object. Londrina, PR, 2006.

\begin{tabular}{lcc}
\hline Objeto Causador & Freqüência & Porcentagem \\
\hline Pérfuro Cortante & 234 & 92,5 \\
Não Pérfuro & 19 & 7,5 \\
Cortante & 253 & 100,0 \\
Total &
\end{tabular}

Outros trabalhos confirmam que, apesar das instituições de saúde terem aumentado a oferta de equipamento de proteção individual, esses profissionais ainda demonstram pouca adesão ao uso desses equipamentos $^{4,15-17}$

No quadro de pessoal das instituições notificadoras, mostrados na Tabela 2 , os auxiliares de enfermagem (39,5\%) é a categoria profissional que mais se acidenta ${ }^{18}$. Esse grupo, além de ser o de maior contingente, são os profissionais que estão em contato direto com o paciente, administrando medicamentos, realizando curativos e outros procedimentos que os mantêm em constante contato com o risco de acidente.

Constatou-se que os auxiliares de serviços gerais $(10,3 \%)$, ao limparem superfícies e pisos e ao recolherem os lixos, encontram objetos perfuro-cortantes descartados em locais inadequados ou a caixa coletora com a capacidade de lixo acima do recomendado $^{19}$.

Cabe considerar que a disponibilidade das caixas coletoras utilizadas para descarte de material perfurante nem sempre são adequadas para esse fim, sendo algumas vezes adaptações de recipientes como caixas de remédios e embalagens de produtos de limpeza ${ }^{20,21}$.

Em seguida, os estagiários aparecem com 7,5\% das ocorrências, devendo-se este fato ao grande número de escolas técnicas e universidades existentes na região de Londrina, onde os alunos estagiam em diversas instituições de saúde, muitas vezes sem condições técnicas ou sem supervisão 
Tabela 2 - Distribuição dos acidentes com exposição a material biológico, segundo a ocupação do profissional. Londrina, PR, 2006.

Table 2 - Distribution of accidents with exposure to biological material by occupation. Londrina, PR, 2006.

\begin{tabular}{lcc}
\hline Ocupação & Freqüência & Porcentagem \\
\hline Agente Comunitário de Saúde & 3 & 1,2 \\
Atendente de Farmácia & 1 & 0,4 \\
Aux Administrativo/Escriturário & 3 & 1,2 \\
Auxiliar de Enfermagem & 100 & 39,5 \\
Auxiliar de Laboratório & 6 & 2,4 \\
Auxiliar de Odontologia & 7 & 2,8 \\
Auxiliar de Lavanderia & 5 & 2,0 \\
Coletor de Lixo & 13 & 5,1 \\
Dentista & 9 & 3,6 \\
Enfermeiro & 9 & 3,6 \\
Estagiário & 19 & 7,5 \\
Farmacêutico/Bioquímico & 4 & 1,6 \\
Fisioterapeuta & 1 & 0,4 \\
Médico & 14 & 5,5 \\
Não Consta & 6 & 2,4 \\
Outros & 4 & 1,6 \\
Policial Militar/Civil/Bombeiro & 6 & 2,4 \\
Serviços Gerais & 26 & 10,3 \\
Técnico de Enfermagem & 15 & 5,9 \\
Técnico de Higiene Dental & 1 & 0,4 \\
Técnico de Laboratório & 1 & 0,4 \\
Total & 253 & 100,0 \\
\hline
\end{tabular}

direta das escolas, ou mesmo indireta dos serviços, devido a alta demanda de ações de saúde existentes ${ }^{5}$.

O número de coletores de lixo $(5,1 \%)$ também chama a atenção, sendo que esta incidência pode ser justificada pelo período de transição/adaptação pelas empresas coletoras quanto à implantação do Plano de Gerenciamento de Resíduos de Saúde, onde ocorreram muitos descartes inadequados de materiais perfuro-cortantes em lixo comum ${ }^{6}$.

Em referência à variável sexo do acidentado, mostrado na Tabela 3, é compreensível encontrarmos $73,5 \%$ de ocorrência dos acidentes no sexo feminino por ser a enfermagem e os serviços gerais categorias majoritariamente femininas, isto é, profissionais historicamente com a maior força de trabalho presente nas instituições de saúde. Esses dados confirmam que a exposição ao risco e aos acidentes são mais freqüentes no sexo feminino ${ }^{22}$.
Tabela 3 - Distribuição dos acidentes com exposição a material biológico, segundo o sexo. Londrina, PR, 2006.

Table 3 - Distribution of accidents with exposure to biological material by gender. Londrina, $P R$, 2006.

\begin{tabular}{lcc}
\hline Sexo & Freqüência & Porcentagem \\
\hline Feminino & 186 & 73,5 \\
Masculino & 67 & 26,5 \\
Total & 253 & 100,0 \\
\hline
\end{tabular}

Na Tabela 4, verifica-se que as Unidades Básicas de Saúde (UBS) são os locais onde mais ocorreram os acidentes $(24,1 \%)$, devido ao grande contingente de trabalhadores locados nessas unidades, seguindo-se as Unidades de Centro Cirúrgico (14,2\%). Em relação à UBS, pesquisa realizada em Unidade de Saúde Pública de Ribeirão Preto, SP, constatou que $46,7 \%$ da população de trabalhadores considerava o ambiente de 
trabalho o fator mais estressante, favorecendo a ocorrência de acidentes ${ }^{23}$. Outro estudo em unidades de Centro Cirúrgico em hospitais do Brasil mostrou que 1.358 profissionais passaram por algum tipo de exposição a material biológico em um período de 12 meses $^{24}$. Vale lembrar que os profissionais mais expostos ao risco, como as equipes de enfermagem e médica, vêm se submetendo a dupla jornada de trabalho, levando-os a um quadro de cansaço, falta de atenção e estresse.

Ainda nesta tabela, constatou-se a grande quantidade de fichas de notificação sem o preenchimento do campo "local onde ocorreu o acidente" $(9,5 \%)$, remetendo a uma recomendação de constante necessidade de treinamento em serviço.

O sangue é o material biológico que tem os maiores títulos de vírus da Hepatite B (HVB) e é o principal responsável pela transmissão do vírus no serviço de saúde. O risco de contaminação pelo HVB está relacionado, principalmente, ao grau de ex- posição ao sangue no ambiente de trabalho e também à presença ou não do antígeno HbeAg no paciente-fonte. Em exposições percutâneas envolvendo sangue, sabidamente infectado pelo HVB e com HbeAg positivo, o risco de hepatite clínica varia entre 22 a $31 \%$. Quando o paciente fonte apresenta somente a presença de HbsAg positivo com HbeAg negativo, o risco de hepatite clínica varia de 1 a $6 \%{ }^{25}$.

A vacina para hepatite $B$, uma das principais medidas de prevenção pré-exposição, é extremamente eficaz com 90 a $95 \%$ de resposta vacinal em adultos imunocompetentes ${ }^{25}$, sendo oferecida gratuitamente na rede pública de saúde desde 1995. Com base nestes dados (Tabela 5), considerou-se o índice de 25,7\% de trabalhadores não vacinados ou com seu estado vacinal ignorado bastante elevado, revelando a necessidade constante de campanhas de vacinação e orientação em serviço.

A partir de 1997, com a implantação da ficha de notificação de acidentes com

Tabela 4 - Distribuição dos acidentes com exposição a material biológico, segundo a unidade de trabalho. Londrina, PR., 2006.

Table 4 - Distribution of accidents with exposure to biological material by work setting. Londrina, $P R$, 2006.

\begin{tabular}{lcc}
\hline Unidade de Trabalho & Freqüência & Porcentagem \\
\hline Ambulatório & 10 & 4,0 \\
Centro Cirúrgico & 36 & 14,2 \\
Centro de Material & 2 & 0,8 \\
Clínica Odontológica & 6 & 2,4 \\
Enfermaria & 21 & 8,3 \\
Farmácia & 4 & 1,6 \\
Laboratório & 10 & 4,0 \\
Lavanderia & 5 & 2,0 \\
Maternidade & 2 & 0,8 \\
MI & 2 & 0,8 \\
Não Consta & 24 & 9,5 \\
Outros & 21 & 8,3 \\
Pediatria & 3 & 1,2 \\
Pronto Socorro & 23 & 9,1 \\
Unidade Básica de Saúde & 61 & 24,1 \\
Unidade Internação de RN & 1 & 0,4 \\
UTI & 7 & 2,8 \\
UTI Neonatal & 2 & 0,8 \\
Via Pública & 13 & 5,1 \\
Total & 253 & 100,0 \\
\hline
\end{tabular}


Tabela 5 - Distribuição dos acidentes com exposição a material biológico, segundo a situação vacinal da vacina contra hepatite $B$. Londrina, PR, 2006.

Table 5 - Distribution of accidents with exposure to biological material by hepatitis $B$ vaccination status. Londrina, PR, 2006.

\begin{tabular}{lcc}
\hline Vacina Hepatite & Freqüência & Porcentagem \\
\hline Vacinado & 188 & 74,3 \\
Ignorado & 65 & 25,7 \\
Total & 253 & 100,0 \\
\hline
\end{tabular}

material biológico, inicia-se timidamente um fluxo de notificações ao Serviço de Epidemiologia da Secretaria Municipal de Saúde. A partir da realização de um seminário em 2005 com todas as instituições empregadoras de Londrina, o fluxo de atendimento do acidente foi reorganizado gerando aumento das fichas de notificação. Como se vê na Tabela 6, a Rede Pública aglutina a maior parte das instituições de saúde e gerou $49,8 \%$ das notificações devido a um melhor acompanhamento e busca ativa dos casos pelo próprio serviço. Entende-se que a partir destes dados, o mesmo processo tem acontecido em outras instituições empregadoras que, na medida em que estão sendo sensibilizadas para atenção ao acidente de trabalho, estão notificando melhor e dando seguimento e acompanhamento ao acidentado.

\section{Considerações Finais}

Em resposta aos números crescentes de acidentes de trabalho com exposição a material biológico, o Ministério da Saúde, junto aos Estados e municípios, tem demonstrado uma preocupação em estruturar os Centros de Referência em Saúde do Trabalhador e disponibilizar treinamentos que subsidiem a prática em saúde nesse campo. Na linha do levantamento de dados este trabalho mostrou que, no que se refere ao acidente com exposição a material biológico, 92,5\% ocorreram com objetos perfuro-cortantes. Do total de notificações analisadas, constatou-se que a categoria auxiliar de enfermagem obteve uma freqüência de 39,5\% dos acidentes, vindo em seguida os serviços gerais $10,3 \%$ e os estagiários com 7,5\%.

Considerando a unidade de trabalho, $24,1 \%$ ocorreram em unidades básicas de saúde, $14,2 \%$ em centro cirúrgico, 9,1\% em pronto socorro e $8,3 \%$ em enfermarias. Pode-se inferir que essas unidades apresentaram maior número de notificações por serem locais onde mais são realizados procedimentos de riscos usando materiais perfuro-cortantes, locais de tensão e muitas vezes de excesso de trabalho, o que pode ocasionar cansaço ou não adesão às normas de biossegurança.

Quanto à situação vacinal, considera-se que, a despeito de o Brasil dispor da vacina gratuitamente desde 1995 e a partir de 1996

Tabela 6 - Distribuição dos acidentes com exposição a material biológico, segundo a instituição empregadora. Londrina, PR, 2006.

Table 6 - Distribution of accidents with exposure to biological materia, by type of employer. Londrina, PR, 2006.

\begin{tabular}{lcc}
\hline Instituição & Freqüência & Porcentagem \\
\hline Rede Pública & 126 & 49,8 \\
Rede Privada Filantrópica & 28 & 11,1 \\
Rede Privada Conveniada & 27 & 10,7 \\
Rede Privada & 33 & 13,0 \\
Outros & 12 & 4,7 \\
Instituição de Ensino & 16 & 6,3 \\
Coleta Lixo/Limpeza/Conservação & 11 & 4,4 \\
Total & 253 & 100,0 \\
\hline
\end{tabular}


para profissionais da saúde, registrou-se $25,7 \%$ de ignorados ou não vacinados, uma expressão da ainda incipiente preocupação dos serviços de saúde em estarem encaminhando seus profissionais para atualizarem seu quadro vacinal.

Em paralelo aos dados levantados, constata-se que o município de Londrina-PR, após a habilitação do CEREST e em parceria com o Serviço de Epidemiologia, tem apontado melhorias no sistema de notificação dos acidentes de trabalho com exposição a material biológico a partir da discussão dos protocolos e readequação dos fluxogramas para o atendimento do profissional acidentado.

Um estudo deste tipo permite considerar igualmente a importância de se abrir espaços para discussões ampliadas envolvendo a equipe multiprofissional de saúde e seus parceiros, como bombeiros, policias militares e civis, coletores de lixo, onde todos possam desvelar no momento próprio suas dúvidas e falar da sobrecarga de trabalho que acompanha o fazer saúde.

\section{Referências}

1. Braga D. Acidente de trabalho com material biológico em trabalhadores da equipe de enfermagem do Centro de Pesquisas Hospital Evandro Chagas [dissertação de mestrado]. Rio de Janeiro: Fundação Oswaldo Cruz, Escola Nacional de Saúde Pública; 2000.

2. Brrasil. Ministério da Saúde. Recomendações para atendimento e acompanhamento de exposição ocupacional a material Biológico: HIV e Hepatites B e C. Brasília: Ministério da Saúde; 2004.

3. Brasil. Lei $\mathrm{n}^{\circ} 8.213$, de 24 de julho de 1991. Dispõe sobre os planos de benefícios da Previdência Social e dá outras providências. Disponível em: http://www6.senado.gov. br/legislacao/ListaTextoIntegral.action [Acessado em: 9 de março de 2006].

4. Caetano JA, Soares E, Braquehais AR, Rolim KAC. Acidentes de trabalho com material biológico no cotidiano da enfermagem em unidade de alta complexidade. Enfermeria Global 2006; 9: 1-10. Disponível em: http://www.um.es/eglobal/9/ pdf/09d02p.pdf [Acessado em: 18 de março de 2007].

5. Toledo Júnior, ACC. et al.. Conhecimento, atitudes e comportamentos frente ao risco ocupacional de exposição ao HIV entre estudantes de Medicina da Faculdade de Medicina da Universidade Federal de Minas Gerais. Rev Soc Bras Med Trop [periódico na Internet] 1999 Out [citado 2007 Out 26] ; 32(5): 509-15. Disponível em: http://www. scielo.br/scielo.php?script=sci_arttext $\&$ pid=S0037$86821999000500007 \& \operatorname{lng}=$ pt \&nrm=iso

6. Velloso MP, Santos EM, Anjos LA. Processo de trabalho e acidentes de trabalho em coletores de lixo domiciliar na cidade do Rio de Janeiro, Brasil. Cad. Saúde Pública [periódico na Internet]. 1997 Out [citado 2007 Out 26] ; 13(4): 693-700. Disponível em: http://www. scielosp.org/scielo.php?script=sci_arttext\&pid=S0102311X1997000400012\&lng=pt\&nrm=iso. doi: $10.1590 /$ S0102-311X1997000400012
7. Brasil. Ministério da Saúde. Secretaria de Ações em Saúde. Departamento de Ações Programáticas Estratégicas. Exposição a materiais biológicos. Brasília: Ministério da Saúde; 2006.

8. Monteiro MS, Carnio AM, Alexandre NMC. Acidentes de trabalho entre o pessoal de enfermagem de um hospital universitário. Rev Bras Enfermagem 1987; 40(2/3): 89-92.

9. Alves NA, Robazzi MLCC, Marziale MHP, Hayashida M. Causas de subnotificação de acidentes do trabalho entre trabalhadores de enfermagem. Rev Latin-am Enfermagem 2000; 8(3): 119-120. Disponível em: http:// www.scielo.br [Acessado em: 18 de março de 2007].

10. Jorge R. et al.. Acidentes biológicos em Hospital Universitário. Rev. Med Hosp São Vicente de Paulo 2000; 11(26): 19-22..

11. Sant'Ana A. Biossegurança no Brasil: a necessidade de uma política consistente. In: Teixeira P, Valle S. (org.) Biossegurança: uma abordagem multidisciplinar. Rio de Janeiro: Editora Fiocruz; 1996. p. 27-40.

12. Sarquis LMM. Acidentes de trabalho com instrumentos pérfuro-cortantes: ocorrência entre os trabalhadores de enfermagem [dissertação de mestrado]. São Paulo: Escola de Enfermagem da USP; 1999.

13. Caetano R, Loja TB, Lima CXB. Quando o risco é nosso: acidentes de trabalho com material biológico entre profissionais do HU Pedro Ernesto-UERJ. In: Anais do $6^{\circ}$ Congresso Brasileiro de Saúde Coletiva (junho de 1999) Salvador (BA). Rio de Janeiro: ABRASCO; 2000. v.5, p. 493.

14. Belei RA, et al.. O impacto do acidente com material biológico na vida de profissionais e alunos de um hospital Universitário. Espaço para Saúde 2001; 2 (2). Disponível em: http://www.ccs.uel.br/ espacoparasaude/v2n2/doc/acidente.doc [Acessado em: 10 de dezembro de 2006]. 
15. Sailer GC. Adesão de trabalhadores de enfermagem ao tratamento com anti-retrovirais pós-exposição ocupacional a material biológico [dissertação de mestrado] Ribeirão Preto: Escola de Enfermagem de Ribeirão Preto; 2004.

16. Balsamo AC, Felli VA. Study of work accidents related to human body fluids exposure among health workers at a university hospital. Rev Latino-am Enfermagem 2006; 14(3): 346-53. Disponível em: www.scielo.br/scielo.br [Acessado em: 18 de março de 2007].

17. Caixeta RB, Barbosa-Branco A. Acidente de trabalho, com material biológico, em profissionais de saúde de hospitais públicos do Distrito Federal, Brasil, 2002/2003. Cad Saúde Pública [periódico na Internet]. 2005 Jun [citado 2007 Out 26] ; 21(3): 737-746. Disponível em: http://www.scielo. br/scielo.php?script=sci_arttext\&pid=S0102$311 X 2005000300007 \& \operatorname{lng}=$ pt\&nrm=iso.

18. Brandão Junior PS. Biossegurança e AIDS: as dimensões psicossociais do acidente com material biológico no trabalho em hospital [dissertação de mestrado]. Rio de Janeiro: Fundação Oswaldo Cruz, Escola Nacional de Saúde Pública; 2002.

19. Rodrigues NML Conhecimento e adesäo às medidas de precauções padrão dos funcionários do serviço de higiene e limpeza hospitalar [dissertação de mestrado]. Säo Paulo: Curso de Enfermagem da Escola Paulista de Medicina da Universidade Federal de São Paulo; 2001.
20. Souza M, Vianna LAC. Incidência de acidentes de trabalho relacionada com a não utilização das precauções universais. Rev Bras Enfermagem 1999; 46(3/4): 234-44.

21. Marziale MHP, Rodrigues CM. A produção científica sobre os acidentes de trabalho com material pérfuro cortante entre trabalhadores de enfermagem. Rev Latino-am Enfermagem 2002; 10(4): 571-77.

22. Conselho Federal de Enfermagem. Estatísticas 2005. Avaliable from: URL: htpp://www.portalcofen.com.br. [cited 2005 jul 20].

23. Chiodi MB, Marziale MHP, Robazzi MLCC. Acidentes de trabalho com material biológico entre trabalhadores de unidades de saúde pública. Rev Latino-Am. Enfermagem [periódico na Internet]. 2007 Ago [citado 2007 Out 26] ; 15(4): 632-638. Disponível em: http://www. scielo.br/scielo.php?script=sci_arttext $\&$ pid=S0104$11692007000400017 \& \ln =$ pt\& nrm=iso.

24. Lacerda RA, Cursino, LC, Silva PB. Exposições ocupacionais a substâncias orgânicas de pacientes em Centro Cirúrgico. Rev Sobecc 2001; 5(5): 24-9.

25. Brasil. Ministério da Saúde. Secretaria de Políticas de Saúde. Coordenação Nacional de DST e Aids. Manual de condutas exposição ocupacional a material biológico: Hepatite e HIV. Brasília: Ministério da Saúde; 1999.

Recebido em: 16/04/07 Versão final reapresentada em: 05/03/08

Aprovado em: 19/03/08 